\title{
3D displacement retrieval on glacial areas by airborne multi-view photogrammetry
}

Haixing HE*, Flavien Vernier ${ }^{\dagger}$, Thierry Villemin*, Estelle Ployon *, Philip Deline* and Umberto Morra di Cella $\neq$

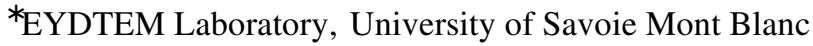

Bâtiment « Pôle Montagne », F-73376 Le Bourget du Lac, France

Email : haixing.he@univ-savoie.fr

${ }^{\dagger}$ LISTIC Laboratory, Polytech Annecy Chambéry

5 chemin de bellevue, France

${ }^{\ddagger}$ ARPA Valle d'Aosta,

Località Grande Charrière, 44, 11020 Saint-Christophe (AO), Italy 
Résumé-With the development of photogrammetry and computer vision, Unmanned Aerial Vehicle (UAV) embedded with digital compact camera plays an important role on gathering geographic information [1]. In this context, a novel method is proposed in this article for monitoring displacement of natural environment by airborne multi-view photogrammetry. The method is implemented for calculating 3D displacement of a glacier area in one month and a half. We illustrate the application of Micmac, a powerful and free photogrammetry software, for point cloud reconstruction. With the GPS embedded on the UAV whose precision is not quiet high, we succeed in georeferencing point clouds. As we do not have any ground control point in land, two different ways of registration have been introduced to align point clouds. Afterwards, a set of tools is presented for calculating 2D displacement maps from point clouds. We introduce at last an inversion processing to calculate a 3D displacement map. The final result shows the 3D displacement of each pixel in the entire scene. Since final result is influenced by the precision of point cloud registration, this paper also highlights useful improvements to do in the future to obtain the 3D displacement with higher precision.

\section{INTRODUCTION}

Nowadays, the temporal evolution of glaciers is often monitored by image acquisition systems. Different observation techniques exist such as aerial photogrammetry using an airborne camera [2] and terrestrial photogrammetry using a digital camera [3]. These two techniques provide 2D displacement measurement in diverse directions according to the geometry of each acquisition, while it is more useful to retrieve 3D displacement in order to interpret deformation fields.

With the aide of existing georeferenced 3D model and GPS, 3D displacement can be estimated by ground-based multi-view photogrammetry [3]. However, to retrieve the 3D displacement, nearly all photogrammetric methods require Ground Control Points (GCPs) to georeference a point cloud and calculate 3D displacement. Because of the complexity of relief, it is not always easy to obtain GCPs. As a result, the retrieval of $3 \mathrm{D}$ displacement by photogrammetry still remains challenging so far.

This paper presents a low-cost methodology to monitor 3D displacement of glacial areas from airborne images. The method is able to detect deformation fields by using two series of images taken from different times. It is evaluated on two series of photos taken by a drone with integrated GPS. No GCP is available on the ground, only images and positions of the drone during the acquisition are available. The data are used for reconstruction of point clouds by using MicMac [4]. Based on two point clouds obtained from different image acquisitions, a virtual camera is created to take ortho-images from these point clouds, ortho-images are then used to calculate 2D displacement maps. The reason why we develop a virtual camera is that we can control camera acquisition positions and camera parameters including intrinsic and extrinsic parameters. As a result, ortho-images can be captured on two point clouds by using one camera at the same positions. Multiple 2D displacement maps are then calculated by multi-correlation. Finally, the 3D displacement is calculated by using inversion technique [5] based on more than three different 2D displacement maps.

\section{STUDY AREA}

The Miage Glacier is one of the principal Italian glaciers of Mont Blanc massif. The lower glacier is a 8-km-long, gently sloping tongue, partly entrenched in a deep, straight valley [6]. Figure 1 shows the localization of study area and a point cloud we constructed. The point cloud of this glacier area can be manually divided into two parts : the northern part is the glacier area covered by supra-glacial debris and an ice-contact lake, the southern part is a fixed area covered by a great deal of vegetation. The whole scene is about $800 \mathrm{~m}$ from the east to the West and $900 \mathrm{~m}$ from the North to the South.
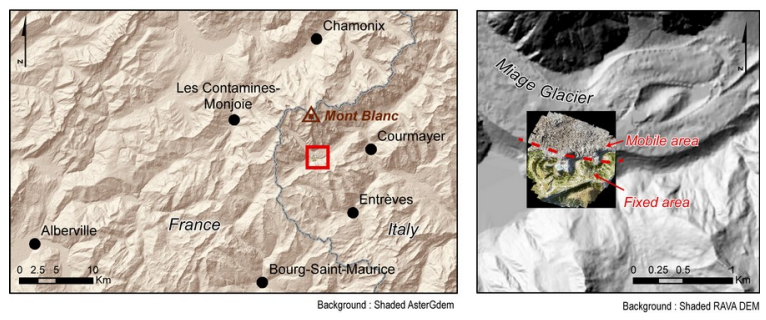

FIGURE 1: Localization of the study area and point cloud reconstruction zone

In this article, the fixed area is used to georeference and align point clouds. Several big rocks along the road were 
particularly selected as characteristic points. The moving area is deformation field where we calculate 3D displacement. Deformation types are not the same and depend on whether the field is glacial area or lake. Glaciers flows towards one direction, therefore its displacement is homogeneous. However, the type of change near the lake is not regular because the level of the lake brings down in summer, more edge areas show up. Since these areas appear only in the latter point cloud, the displacement is not able to calculate.

\section{DATA ACQUISITION}

Two series of images were taken by an UAV from the Miage glacier and lake. Between these two acquisitions, there is an interval of 7 weeks during which changes occurred. For example, the fall of ice pieces into the lake from the northern wall is a quite frequent event [7].

In this study the Swinglet CAM (SC) from SenseFly is used as an unmanned vehicle (UAV). The SC is a fixedwing UAV with $80 \mathrm{~cm}$ wingspan and a maximum take-off weight of $0,550 \mathrm{Kg}$. The nominal endurance is 30 minutes of flight time, the propeller is an electric brushless motor and the nominal cruise speed is $11 \mathrm{~m} / \mathrm{s}(40 \mathrm{Km} / \mathrm{h})$. The SC is mounted with a GPS receiver, wind meter and Canon IXUS 125HS 16 Mp digital compact camera, self-powered and electronically integrated and controlled. The camera is controlled by the autopilot system so as to have images at a correct position. The flights purpose was a piloting training and flight safety check up in operational conditions (altitude, wind, distance between take-off location and survey area). Therefore no GCP was collected.

The first acquisition was taken on 18th July 2014. 143 photos captured by the UAV at an altitude of $170 \mathrm{~m}$ above the ground were recorded in a linear way parallel to the land surface. All the images are in JPEG format with a size of 4608 * 3456 pixels. The second acquisition was carried out on 14th September 2014, we used the same UAV to take 250 pictures. Both flights have a side overlap of $80 \%$ and forward overlap of $85 \%$. More UAV flight features is shown in table I.

\begin{tabular}{|l||r|r|}
\hline & First Flight & Second Flight \\
\hline \hline Start time & $10 \mathrm{~h} \mathrm{47}$ & $8 \mathrm{~h} 22$ \\
\hline Duration & $18 \mathrm{~min}$ & $24 \mathrm{~min}$ \\
\hline number of photo & 143 & 250 \\
\hline Area covered & $0,36 \mathrm{Km}^{2}$ & $0,36 \mathrm{~km}^{2}$ \\
\hline Distance & $14,0 \mathrm{Km}$ & $14,1 \mathrm{Km}$ \\
\hline Altitude above the ground & $170 \mathrm{~m}$ & $170 \mathrm{~m}$ \\
\hline Resolution & $5,0 \mathrm{~cm} / \mathrm{px}$ & $5,0 \mathrm{~cm} / \mathrm{px}$ \\
\hline
\end{tabular}

TABLE I: UAV Acquisition features

\section{3D DISPLACEMENT RETRIEVAL METHOD}

The method of 3D displacement retrieval includes 3 parts : 3D dense point cloud reconstruction, 2D displacement detection and 3D displacement reconstruction. Details of each part can be find in figure 2 .

For the surface reconstruction, Micmac is used to extract the tie-points (Tapioca), calibrate the camera (Tapas), georeference the point cloud (CenterBascule) and compute the dense matching (Malt). Micmac is a set of photogrammetric software that allows to compute a point cloud from a set of images [4].

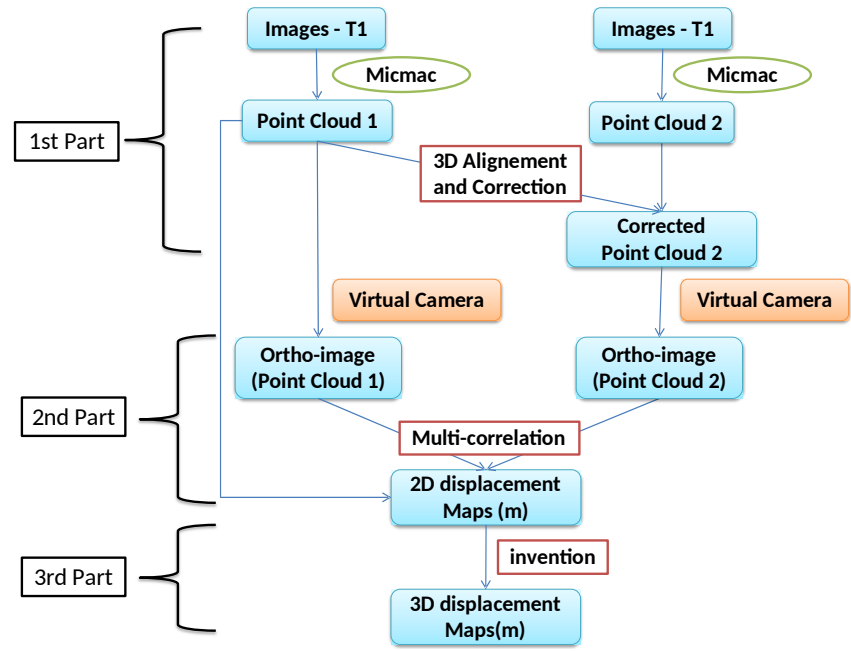

FIGURE 2: Method diagram to retrieve the $3 D$ displacement measurement

By using Micmac, one point cloud was constructed for each series of images.

After the 3D reconstruction, we developed a set of tools for calculating 2D displacement maps. Firstly, georeferencing error of $3 \mathrm{D}$ reconstruction is corrected. Although the 3D reconstruction is georeferenced, an alignment step is required. This is mainly due to the imprecision of camera shooting position given by GPS embedded on UAV. Usually this step requires real GCPs or relative GCPs on the motion free area of one of the 3D reconstructions. Secondly, a virtual camera was created in order to take ortho-images from point clouds. As all intrinsic and extrinsic parameters can be manually set, the visual camera can be put in every location with any orientation. In this article, visual camera has been put in five different positions as shown in figure 3 . Thirdly, at each position, the

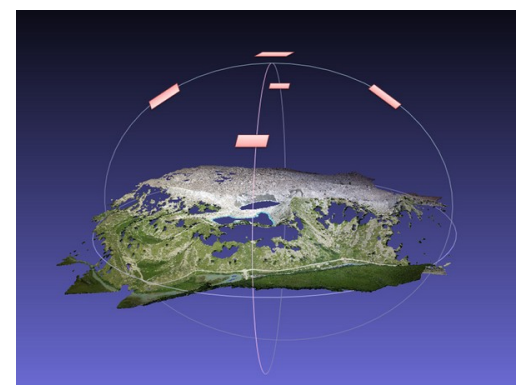

FIGURE 3: Visual camera in five shooting positions

camera takes an ortho-image above each point cloud. As a result, ten ortho-images were obtained from the two point clouds at the five camera positions. We name two ortho-images taken at the same camera position from two different point clouds "a pair". Finally, we obtain a 2D displacement map by correlation from each pair.

Once more than 3 maps of 2D displacement are calculated, a 3D displacement map can be extracted by using inversion technique [5]. To clarify this point further, 2D displacement can be considered as projections of 3D displacement (with 3 
components, East, North, Up) in different directions depending on the image acquisition geometry. Consequently, the 3D displacement can be retrieved from at least three 2D displacement maps in different directions by inversion in the least square sense. For this, projection vectors that link 2D displacement and 3D displacement are necessary. As we already have five 2D displacement maps, we are able to retrieve the 3D displacement field over Miage glacier.

\section{RESULTS}

\section{A. $3 D$ reconstruction and alignment}

After the MicMac processing, we obtain two point clouds, one for each series of images. The first one is that of the Miage Glacier on 18th July 2014. For this cloud, we have about 1300 points $/ \mathrm{m}^{2}$ and we find gaps in areas covered by vegetation or water. The second one is Miage Glacier on 14th September 2014. Since we have more pictures for this series, the density is 1700 points $/ \mathrm{m}^{2}$. As in the first cloud, there are also areas with gaps. This phenomenon is due to changes of vegetation and a lack of texture on the lake surface, both of these situations may cause bad images correlations. To georeference these two point clouds, the GPS embedded on the UAV is used. It records camera's position at each shooting time.

Then, we picked several rocks in the South part of the point cloud as reference points. These rocks are located in the fixed area and have not moved. These reference points are used to compare the difference of fixed area between both point clouds. After comparing 7 points in fixed area, differences are between 0 and $1 \mathrm{~m}$ in $\mathrm{X}$ axe, between 2 and $3 \mathrm{~m}$ in $\mathrm{Y}$ axe and between 6 and $10 \mathrm{~m}$ in $\mathrm{Z}$ axe. These results are not quite ideal because the fixed area has not moved so that differences should be close to zero. The reasons why we have a high difference between both point clouds are the following : firstly, as we have no GCPs on land surface, the georeferencing task is carried out by using embedded GPS of the UAV which has a low precision. Secondly, Micmac also produces errors in the point cloud construction procedure [8].

Next, we tried two different ways to reduce differences in the fixed area between both point clouds. The first one is a point clouds alignment by using CloudCompare. To do so, we separated fixed and moving area for each point cloud and aligned only fixed areas. After comparison, differences in $\mathrm{X}, \mathrm{Y}$ and $\mathrm{Z}$ axes are less than $0.5 \mathrm{~m}$ in fixed area. Then, we applied the transformation matrix onto the whole second point cloud and compared results. In the moving area, a few homologous points were picked to compare their coordinates. The result shows that the difference is less than $0.5 \mathrm{~m}$ in $\mathrm{X}$ and $\mathrm{Y}$ axes. However, it is about $5 \mathrm{~m}$ in $\mathrm{Z}$ axe at the border. The reason is that in moving area, no GCP is available so that the northern part of the point cloud has deviated when it was built. The second method consists in extracting GCP from fixed area of the first point cloud and to use these GCP to reconstruct the second point cloud. After reconstruction, we compared the new point cloud with the first one. The result obtained is very close to the aligned point cloud of the first method.

\section{B. $2 D$ displacement map}

Once we have two well aligned point clouds, a virtual camera is placed into the $3 \mathrm{D}$ environment and takes pictures of both point clouds as shown in figure 3. Five ortho-images have been captured from each point cloud. For instance, figure 4 shows an ortho-image of the first point cloud taken in initial camera position and figure 5 shows an ortho-image of the second point cloud taken in the same camera position.

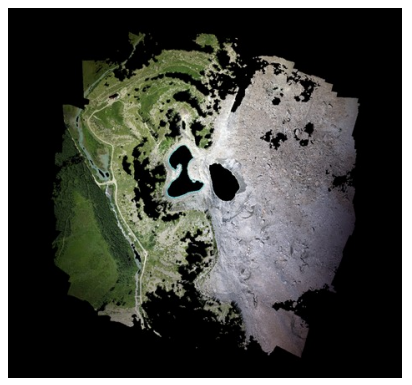

FIGURE 4: Virtual ortho-image of the first point cloud.

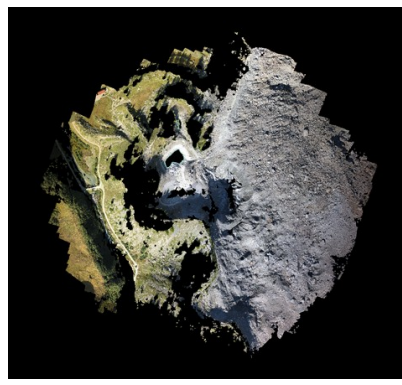

FIGURE 5: Virtual ortho-image of the second point cloud.
Afterwards, the camera is moved to take pictures of the point cloud from four different points of view. These positions are all defined with 20 degrees of angle with the vertical axe $\mathrm{Z}$ (nadir to zenith) and 0, 90, 180 and 270 degrees around the $\mathrm{Z}$ axe. As a consequence, 5 pairs of orthoimages have been captured.

Then, image correlation is applied for each pair and we can get 5 maps of 2D displacement. Figure 6 shows one of the five maps of displacement. For this map, the camera is in the initial position ( 0 degree with $\mathrm{Z}$ and 0 degree around $\mathrm{Z}$ ). The $2 \mathrm{D}$ displacement computed in pixel unit by the correlation algorithm is converted in meters using the georeferenced point cloud. This result is filtered to eliminate most of the inconsistent errors. In this figure, arrows have

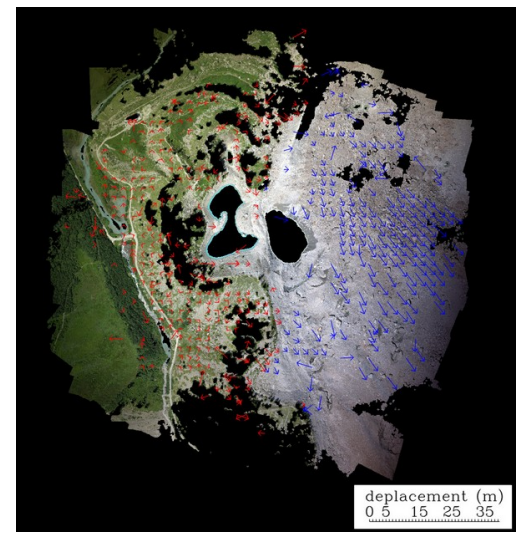

FIGURE 6: Projection of the $2 D$ displacement map onto the ortho-image

been added in order to show the direction and distance of the displacement of each pixel. The direction of arrows is the direction of displacement and the length of arrows stands for the distance of displacement. We can see in figure 6 that in fixed area, the length of red arrows is very short - most of them are less than $0.5 \mathrm{~m}$ - which means that few movement occured. These red arrows illustrate the residual error after alignment. In the moving area, directions of blue arrows are homogeneous 
and meet the direction of movement of the lower part of the Miage glacier (from West to East). For the entire area, we can find that red arrows are more than $0.5 \mathrm{~m}$ and inconsistent blue arrows correspond to correlation errors due to the vegetation or light effects.

\section{C. $3 D$ displacement map}

In order to compute 3D displacement for each pixel, the least squares inversion algorithm is used to fuse 2D displacement maps. The forward equation is given by :

$$
R=P U
$$

Where $P$ is projection matrices, $U$ is the vector of $3 \mathrm{D}$ displacement and $R$ is the $2 \mathrm{D}$ displacement depending on projection matrices. In our case, $P$ and $R$ are known. $P$ is computed according to the positions of the virtual camera and $R$ is the result of correlation algorithm. The inverse problem - to compute $U$ depending on $P$ and $R$ - can be solved by the least squares inversion algorithm. In this case, $U$ is given by :

$$
U=\left(P^{T} P\right)^{-1} P^{T} R,
$$

where $P^{T}$ is the transposed matrix of $P$. As a result, a 3D displacement map is calculated as shown in figure 7 .

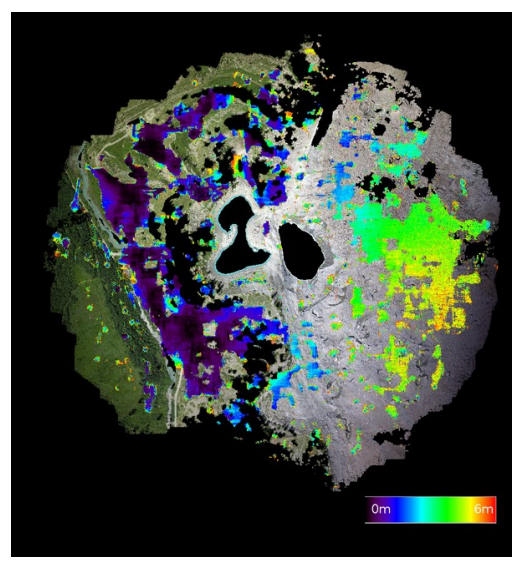

FIGURE 7: 3D displacement amplitude mapped onto the ortho-image
From this figure, we can see the area where the 3D displacement was calculated. Displacement on the fixed area is close to 0 . This result highlights a correct alignment of point clouds. On the glacier, displacement is between 3 and $5 \mathrm{~m}$ which is over estimated, the real displacement is close to $3 \mathrm{~m}$. This error is due to an error on the $\mathrm{Z}$ axe displacement that corresponds to a deviation of alignment on the moving area where there is no GCP.

\section{CONCLUSION}

The lack of GCPs led to a over estimated displacement of two 3D point clouds. In order to obtain a higher precision, higher accuracy point clouds are needed. In general terms, the accuracy of a photo-based point cloud depends on the scale and resolution of the input images, the distribution and accuracy of the control data (ground control points, scale measurements or camera positions), the precision and distribution of the matching image points, and the network geometry, which includes the number of photos, how much they overlap and how convergent the views are [9]. If the surveyed area is easy to access, high precision GCP is recommended. Otherwise, it is better to raise the precision of GPS integrated on the UAV. In case neither of these two ways are possible, then at the moment of image acquisition, we can extract control points from an existing digital elevation model.

In conclusion, this paper offers the latest information regarding the 3D displacement detection by airborne multi-view photogrammetry. The method proposed in this paper is able to identify the $2 \mathrm{D}$ and $3 \mathrm{D}$ displacement of each pixel. Compared to others, this method requires only a GPS integrated drone for acquisition and offers a high precision 3D displacement map. During the procedures of data treatment, all used software and tools are open-source or free of charge, which strongly reduces the cost of the monitoring of glacial areas or landslides deformation. What's more, the method presented in this paper can not only be applied on airborne images but also be applied on optical satellite or terrestrial images with GPS. 


\section{RÉFÉRENCES}

[1] J. T. Sebastian Siebert, "Mobile 3d mapping for surveying earthwork projects using an unmanned aerial vehicle (uav) system," Automation in Construction, pp. 1-14, 2014.

[2] S. P. Bemis and S. Micklethwaite, "Ground-based and uav-based photogrammetry: A multi-scale, high-resolution mapping tool for structural geology and paleoseismology," Journal of Structural Geology, vol. 69, pp. 163-178, 2014.

[3] J. Travelletti, C. Delacourt, P. Allemand, J.-P. Malet, J. Schmittbuhl, R. Toussaint, and M. Bastard, "Correlation of multi-temporal groundbased optical images for landslide monitoring: Application, potential and limitations," ISPRS Journal of Photogrammetry and Remote Sensing, pp. 39-55, 2012.

[4] M. Deseilligny, J. Beleveau, G. Choqueux, M. Deveau, and L. Girod, MicMac, Apero and Other Beverages in a Nutshell, 2013.

[5] Y. Yan, "Fusion de mesures de déplacement issues d'imagerie sar : application aux modélisations séismo-volcaniques," Ph.D. dissertation, Laboratoire LISTIC et ISTERRE， 2011.

[6] P. Deline and G. Orombelli, "Glacier fluctuations in the western alps during the neoglacial, as indicate by the miage morainic amphitheatre (mont blanc massif, italy)," BOREAS, pp. 456-467, 2005.

[7] S. Tinti, A. Maramai, and A. V. Cerutti, "The miage glacier in the valley of aosta (western alps, italy) and the extraordinary detachment which occurred on august 9,1996," Pergamon, vol. 24, pp. 157-161, 1999.

[8] A. Strumpf, J.-P. Malet, P. Allemand, M. Pierrot-Deseilligny, and G. Skupinski, "Ground-based multi-view photogrammetry for the monitoring of landslide deformation and erosion," Geomorphology, pp. 130-145, 2014.

[9] S. P. Bemis, S. Micklethwaite, D. Turner, M. R. James, S. Akciz, S. T. Thiele, and H. A. Bangash, "Ground-based and uav-based photogrammetry : A multi-scale, high resolution mapping tool for structural geology and paleoseismology," Journal of Structural Geology, pp. 163-178, 2014. 